\title{
Killing Vector Fields in Generalized Conformal $\beta$-Change of Finsler Spaces
}

\author{
Mallikarjun Yallappa Kumbar, ${ }^{1}$ Narasimhamurthy Senajji Kampalappa, ${ }^{2}$ \\ Thippeswamy Komalobiah Rajanna, ${ }^{2}$ and Kavyashree Ambale Rajegowda ${ }^{2}$ \\ ${ }^{1}$ Department of P.G. Mathematics, Vijaya College, Rashtriya Vidyalaya Road, Basavanagudi, Bangalore, Karnataka 560004, India
${ }^{2}$ Department of P.G. Studies and Research in Mathematics, Kuvempu University, Shankaraghatta, Shimoga, Karnataka 577451, India
}

Correspondence should be addressed to Mallikarjun Yallappa Kumbar; mallikarjunykumbar@gmail.com

Received 20 July 2015; Accepted 4 October 2015

Academic Editor: Ljubisa Kocinac

Copyright (C) 2015 Mallikarjun Yallappa Kumbar et al. This is an open access article distributed under the Creative Commons Attribution License, which permits unrestricted use, distribution, and reproduction in any medium, provided the original work is properly cited.

\begin{abstract}
We consider a Finsler space equipped with a Generalized Conformal $\beta$-change of metric and study the Killing vector fields that correspond between the original Finsler space and the Finsler space equipped with Generalized Conformal $\beta$-change of metric. We obtain necessary and sufficient condition for a vector field Killing in the original Finsler space to be Killing in the Finsler space equipped with Generalized Conformal $\beta$-change of metric.
\end{abstract}

\section{Introduction}

In 1976, Hashiguchi [1] studied the conformal change of Finsler metrics; namely, $\bar{L}=e^{\sigma(x)} L$. In particular, he also dealt with the special conformal transformation named $C$ conformal transformation. This change has been studied by Izumi [2] and Kropina [3]. In 2008, Abed [4, 5] introduced the transformation $\bar{L}=e^{\sigma(x)} L+\beta$, thus generalizing the conformal, Randers, and generalized Randers changes. Moreover, he established the relationships between some important tensors associated with $(M, L)$ and the corresponding tensors associated with $(M, \bar{L})$. He also studied some invariant and $\sigma$-invariant properties and obtained a relationship between the Cartan connection associated with $(M, L)$ and the transformed Cartan connection associated with $(M, \bar{L})$.

In this paper, we deal with a general change of Finsler metrics defined by

$$
L(x, y) \longrightarrow \bar{L}(x, y)=f\left(e^{\sigma(x)} L(x, y), \beta(x, y)\right),
$$

where $f$ is a positively homogeneous function of degree one in $\bar{L}:=e^{\sigma} L$ and $\beta$. This change will be referred to as a generalized $\beta$-conformal change. It is clear that this change is a generalization of the abovementioned changes and deals simultaneously with $\beta$-change and conformal change. It combines also the special case of Shibata $(\bar{L}=f(L, \beta))$ and that of Abed $\left(\bar{L}=e^{\sigma} L, \beta\right)$.

In 1984, Shibata [6] studied $\beta$-change of Finsler metrics and discussed certain invariant tensors under such a change. Killing equations play important role in the study of a Finsler space which undergoes a change in the metric. In fact, they give an equivalent characterization for the transformations to preserve distances. In 1979, Singh et al. [7] studied a Randers space $F^{n}\left(M, L(x, y)=\left(g_{i j}(x) y^{i} y^{j}\right)^{1 / 2}+b_{i}(x) y^{i}\right), n \geq 2$, which undergoes a change $L(x, y) \mapsto L^{*}(x, y)=L^{2}(x, y)+$ $\left(\alpha_{i}(x) y^{i}\right)^{2}$. They discussed Killing correspondence of spaces $F^{n}(M, L)$ and $F^{* n}\left(M, L^{*}\right)$.

In the present paper, we consider a general Finsler space $F^{n}(M, L)$ which undergoes conformal and $\beta$-change; that is, $L(x, y) \rightarrow \bar{L}(x, y)=f\left(e^{\sigma(x)} L(x, y), \beta(x, y)\right)$, where $\beta(x, y)=b_{i}(x) y^{i}$ is a 1 -form. We study Killing correspondence of Finsler spaces $F^{n}(M, L)$ and $\bar{F}^{n}(M, \bar{L})$. For the notations and terminology, we refer the reader to the books $[8,9]$ and the papers [6] by Shibata and [10] by Youssef et al.

\section{Preliminaries}

Let $F^{n}=(M, L), n \geq 2$, be an $n$-dimensional $C^{\infty}$ Finsler manifold with fundamental function $L=L(x, y)$. Consider 
the following change of Finsler structures which will be referred to as a generalized $\beta$-conformal change:

$$
L(x, y) \longrightarrow \bar{L}(x, y)=f\left(e^{\sigma(x)} L(x, y), \beta(x, y)\right) \text {, }
$$

where $f$ is a positively homogeneous function of degree one in $e^{\sigma} L$ and 1 -form $\beta$, where $\beta=b_{i}(x) d x^{i}$.

We define

$$
\begin{aligned}
f_{1} & :=\frac{\partial f}{\partial \widetilde{L}} \\
f_{2} & :=\frac{\partial f}{\partial \beta} \\
f_{12} & :=\frac{\partial^{2} f}{\partial \widetilde{L} \partial \beta}, \ldots,
\end{aligned}
$$

where $\widetilde{L}=e^{\sigma} L$. [10]

The angular metric tensor $\bar{h}_{i j}$ of the space $\bar{F}^{n}$ is given by

$$
\bar{h}_{i j}=e^{\sigma} p h_{i j}+q_{0} m_{i} m_{j}
$$

where

$$
\begin{aligned}
p & =\frac{f f_{1}}{L}, \\
q & =f f_{2}, \\
q_{0} & =f f_{22}, \\
p_{0} & =f_{2}^{2}+q_{0}, \\
q_{-1} & =\frac{f f_{12}}{L}, \\
p_{-1} & =q_{-1}+\frac{p f_{2}}{f}, \\
q_{-2} & =\frac{f\left(e^{\sigma} f_{11}-f_{1} / L\right)}{L^{2}}, \\
p_{-2} & =q_{-2}+\frac{e^{\sigma} p^{2}}{f^{2}}, \\
m_{i} & =b_{i}-\frac{\beta y^{i}}{L^{2}} \neq 0, \\
\sigma_{i} & =\partial_{i} \sigma .
\end{aligned}
$$

$h_{i j}$ is the angular metric tensor of $F^{n}$. The fundamental metric tensor $\bar{g}_{i j}$ and its inverse $\bar{g}^{i j}$ of $\bar{F}^{n}$ are expressed as [10]

$$
\begin{aligned}
\bar{g}_{i j}= & e^{\sigma} p g_{i j}+p_{0} b_{i} b_{j}+e^{\sigma} p_{-1}\left(b_{i} y_{j}+b_{j} y_{i}\right) \\
& +e^{\sigma} p_{-2} y_{i} y_{j}, \\
\bar{g}^{i j}= & \left(\frac{e^{-\sigma}}{p}\right) g^{i j}-s_{0} b^{i} b^{j}-s_{-1}\left(b^{i} y^{j}+b^{j} y^{i}\right)-s_{-2} y^{i} y^{j},
\end{aligned}
$$

where

$$
\begin{aligned}
s_{0} & =\frac{e^{-\sigma} f^{2} q_{0}}{\left(\varepsilon p L^{2}\right)}, \\
s_{-1} & =\frac{p_{-1} f^{2}}{\left(\varepsilon p L^{2}\right)}, \\
s_{-2} & =\frac{p_{-1}\left(e^{\sigma} m^{2} p L^{2}-b^{2} f^{2}\right)}{\left(\varepsilon p \beta L^{2}\right)}, \\
\varepsilon & =\frac{f^{2}\left(e^{\sigma} p+m^{2} q_{0}\right)}{L^{2}} \neq 0, \\
m^{2} & =g^{i j} m_{i} m_{j} .
\end{aligned}
$$

$g_{i j}$ and $g^{i j}$, respectively, are the metric tensor and inverse metric tensor of $F^{n}$. The Cartan tensor $\bar{C}_{i j k}$ and the associate Cartan tensor $\bar{C}_{i j}^{l}$ of $\bar{F}^{n}$ are given by the following expressions:

$$
\begin{aligned}
\bar{C}_{i j k}= & e^{\sigma} p C_{i j k}+\frac{1}{2} e^{\sigma} p_{-1}\left(h_{i j} m_{k}+h_{j k} m_{i}+h_{k i} m_{j}\right) \\
& +\frac{1}{2} p_{02} m_{i} m_{j} m_{k} .
\end{aligned}
$$

The $(h) h v$-torsion tensor $\bar{C}_{i j}^{l}$ is expressed in terms of $C_{i j}^{l}$ as [10]

$$
\bar{C}_{i j}^{l}=C_{i j}^{l}+M_{i j}^{l}
$$

where

$$
\begin{aligned}
M_{i j}^{l} & =\frac{1}{2 p}\left[e^{-\sigma} m^{l}-p m^{2}\left(s_{0} b^{l}+s_{-1} y^{l}\right)\right] \\
& \cdot\left(e^{\sigma} p_{-1} h_{i j}+p_{02} m_{i} m_{j}\right)-e^{\sigma}\left(s_{0} b^{l}+s_{-1} y^{l}\right) \\
& \cdot\left(p C_{i s j} b^{s}+p_{-1} m_{i} m_{j}\right)+\frac{p_{-1}}{2 p}\left(h_{i}^{l} m_{j}+h_{j}^{l} m_{i}\right) ; \\
h_{j}^{i} & =g^{i l} h_{l j}, \\
p_{02} & =\frac{\partial p_{0}}{\partial \beta} .
\end{aligned}
$$

$C_{i j k}$ and $C_{i j}^{l}$ are, respectively, the Cartan tensor and the associate Cartan tensor of $F^{n}$. The spray coefficients $\bar{G}^{i}$ of $\bar{F}^{n}$ in terms of the spray coefficients $G^{i}$ of $F^{n}$ are expressed as [10]

$$
\bar{G}^{i}=G^{i}+D^{i}
$$


where

$$
\begin{aligned}
D^{i} & =\frac{\sigma_{0}}{2 p}\left\{\left[2 p-\beta p_{-1}-e^{\sigma} p^{2} L^{2} s_{-2}\right.\right. \\
& \left.\left.-p s_{-1}\left(2 e^{\sigma} p \beta+e^{\sigma} p_{-1} L^{2} m^{2}\right)\right] y^{i}-2 e^{\sigma} p^{2} \beta s_{0} b^{i}\right\} \\
& +\frac{q}{p} e^{-\sigma} F_{0}^{i}-\frac{1}{2} L^{2} \sigma^{i}+\frac{1}{2}\left(e^{\sigma} p E_{00}-2 q F_{\beta 0}\right. \\
& \left.+e^{\sigma} p L^{2} \sigma_{\beta}\right)\left(s_{0} b^{i}+s_{-1} y^{i}\right) ; \\
E_{j k} & =\left(\frac{1}{2}\right)\left(b_{j \mid k}+b_{k \mid j}\right), \\
F_{j k} & =\left(\frac{1}{2}\right)\left(b_{j \mid k}-b_{k \mid j}\right), \\
F_{j}^{i} & =g^{i k} F_{k j} .
\end{aligned}
$$

The symbol "|" denotes $h$-covariant derivative with respect to Cartan connection $C \Gamma$ and lower index " 0 " (except in $s_{0}$ ) denotes the contraction by $y^{i}$.

The relation between the coefficients $\bar{N}_{j}^{i}$ of Cartan nonlinear connection in $\bar{F}^{n}$ and the coefficients $N_{j}^{i}$ of the corresponding Cartan nonlinear connection in $F^{n}$ is given by [10]

$$
\bar{N}_{j}^{i}=N_{j}^{i}+D_{j}^{i}
$$

where

$$
\begin{aligned}
D_{j}^{i} & =\frac{e^{-\sigma}}{p} A_{j}^{i}-\left(s_{0} b^{i}+s_{-1} y^{i}\right) A_{t j} b^{t}-\left(q b_{0 \mid j}\right. \\
& \left.+e^{\sigma} p L^{2} \sigma_{j}\right)\left(s_{-1} b^{i}+s_{-2} y^{i}\right) ; \\
A_{i j} & =E_{00} B_{i j}+F_{i 0} Q_{j}+q F_{i j}+E_{j 0} Q_{i}-2\left(e^{\sigma} p C_{s i j}\right. \\
& \left.+V_{s i j}\right) D^{s}+\frac{1}{2} \sigma_{0}\left[2 e^{\sigma} p g_{i j}+2 e^{\sigma} p_{-1} m_{j} y_{i}-2 \beta B_{i j}\right. \\
& \left.+e^{\sigma} p_{-1}\left(b_{i} y_{j}-b_{j} y_{i}\right)\right]-\frac{1}{2} \sigma_{i}\left(e^{\sigma} L^{2} p_{-1} m_{j}\right. \\
& \left.+2 e^{\sigma} p y_{j}\right)+\frac{1}{2} \sigma_{j}\left(2 e^{\sigma} p y_{i}+e^{\sigma} L^{2} p_{-1} m_{i}\right) ; \\
A_{j}^{i} & =g^{l i} A_{l j}, \\
2 B_{i j} & =e^{\sigma} p_{-1} h_{i j}+p_{02} m_{i} m_{j}, \\
Q_{i} & =e^{\sigma} p_{-1} y_{i}+p_{0} b_{i} .
\end{aligned}
$$

The coefficients $\bar{F}_{j k}^{i}$ of Cartan connection $C \bar{\Gamma}$ in $\bar{F}^{n}$ and the coefficients $F_{j k}^{i}$ of the corresponding Cartan connection $C \Gamma$ in $F^{n}$ are related as follows [10]:

$$
\bar{F}_{j k}^{i}=F_{j k}^{i}+D_{j k}^{i}
$$

where

$$
\begin{aligned}
& D_{j k}^{i}=\left\{\left(\frac{e^{-\sigma}}{p}\right) g^{i t}-\left(s_{0} b^{i}+s_{-1} y^{i}\right) b^{t}-\left(s_{-1} b^{i}\right.\right. \\
& \left.\left.+s_{-2} y^{i}\right) y^{t}\right\}\left\{F_{t k} Q_{j}+F_{t j} Q_{k}+E_{j k} Q_{t}+\frac{1}{2}\right. \\
& \cdot \Theta_{(j, k, t)}\left(2 e^{\sigma} p C_{j k m} D_{t}^{m}+2 V_{j k m} D_{t}^{m}-K_{j k} \sigma_{t}\right. \\
& \left.\left.\quad-2 B_{j k} b_{0 \mid t}\right)\right\}, \\
& V_{i j k}=\frac{1}{2} e^{\sigma} p_{-1}\left(h_{i j} m_{k}+h_{j k} m_{i}+h_{k i} m_{j}\right)+\frac{1}{2} \\
& \cdot p_{02} m_{i} m_{j} m_{k}, \\
& K_{i j}=A_{1} g_{i j}+A_{2} b_{i} b_{j}+A_{3}\left(b_{i} y_{j}+b_{j} y_{i}\right)+A_{4} y_{i} y_{j}, \\
& A_{1}=e^{\sigma}\left(2 p-\beta p_{-1}\right), \\
& A_{2}=-\beta p_{02}, \\
& A_{3}=e^{\sigma} p_{-1}+\left(\frac{\beta^{2}}{L^{2}}\right) p_{02}, \\
& A_{4}=e^{\sigma} p_{-2}-\left(\frac{\beta^{3}}{L^{4}}\right) p_{02}, \\
& \Theta_{(j, k, t)}\left\{A_{j k t}\right\}=A_{j k t}-A_{k t j}-A_{t j k} .
\end{aligned}
$$

The tensor $D_{j k}^{i}$ has the properties

$$
\begin{aligned}
& D_{j 0}^{i}=B_{j 0}^{i}=D_{j}^{i} ; \\
& D_{00}^{i}=2 D^{i},
\end{aligned}
$$

where $B_{j k}^{i}=\partial_{k} D_{j}^{i}$.

\section{Killing Vector Fields in \\ Correspondence of $F^{n}$ and $\bar{F}^{n}$}

Let us consider an infinitesimal transformation

$$
x^{i}=x^{i}+\epsilon v^{i}(x),
$$

where $\epsilon$ is an infinitesimal constant and $v^{i}(x)$ is a contravariant vector field.

The vector field $v^{i}(x)$ is said to be a Killing vector field in $F^{n}$ if the metric tensor of the Finsler space with respect to the infinitesimal transformation (18) is Lie invariant; that is,

$$
\mathfrak{E}_{v} g_{i j}=0,
$$

with $\mathfrak{E}_{v}$ being the operator of Lie differentiation. Equivalently, the vector field $v^{i}(x)$ is Killing in $F^{n}$ if

$$
v_{i \mid j}+v_{j \mid i}+2 C_{i j}^{l} v_{l \mid 0}=0,
$$

where $v_{i}=g_{i l} v^{l}$. 
Now, we prove the following result which gives a necessary and sufficient condition for a Killing vector field in $F^{n}$ to be Killing in $\bar{F}^{n}$.

Theorem 1. A Killing vector field $v^{i}(x)$ in $F^{n}$ is Killing in $\bar{F}^{n}$ if and only if

$$
\begin{gathered}
M_{i j}^{l} v_{l \mid 0}+C_{r j t} v^{t} D_{i}^{r}+C_{r i t} v^{t} D_{j}^{r}+v_{r} D_{i j}^{r} \\
+\bar{C}_{i j}^{l}\left(2 C_{r l t} v^{t} D^{r}+v_{r} D_{l}^{r}\right)=0
\end{gathered}
$$

where $\bar{C}_{i j}^{l}$ is the associate Cartan tensor of $\bar{F}^{n}$.

Proof. Assume that $v^{i}(x)$ is Killing in $F^{n}$. Then (20) is satisfied. By definition, the $h$-covariant derivatives of $v_{i}$ with respect to $C \bar{\Gamma}$ and $C \Gamma$ are, respectively, given as

$$
\begin{aligned}
& \text { (a) } v_{i \| j}=\partial_{j} v_{i}-\left(\dot{\partial}_{r} v_{i}\right) \bar{G}_{j}^{r}-v_{r} \bar{F}_{i j}^{r}, \\
& \text { (b) } v_{i \mid j}=\partial_{j} v_{i}-\left(\dot{\partial}_{r} v_{i}\right) G_{j}^{r}-v_{r} F_{i j}^{r},
\end{aligned}
$$

where $\partial_{j}=\partial / \partial x^{j}$ and " $\|$ " denote the $h$-covariant differentiation with respect to $C \bar{\Gamma}$. Equation $(22)(a)$, by virtue of (11), (15), and (22)(b), takes the form

$$
v_{i \| j}=v_{i \mid j}-2 C_{r i t} v^{t} D_{j}^{r}-v_{r} D_{i j}^{r} .
$$

Now, from (23), we have

$$
\begin{aligned}
v_{i \| j}+v_{j \| i}+2 \bar{C}_{i j}^{l} v_{l \| 0}= & v_{i \mid j}+v_{j \mid i}+2 C_{i j}^{l} v_{l \mid 0}-2 C_{r i t} v^{t} D_{j}^{r} \\
& -2 C_{r j t} v^{t} D_{i}^{r}-2 v_{r} D_{i j}^{r} \\
& -2 \bar{C}_{i j}^{l}\left(2 C_{r l t} v^{t} D^{r}+v_{r} D_{l}^{r}\right) .
\end{aligned}
$$

Using (9) in (24) and applying (20), we get

$$
\begin{aligned}
v_{i \| j}+v_{j \| i}+2 \bar{C}_{i j}^{l} v_{l \| 0}= & 2 M_{i j}^{l} v_{l \mid 0}-2 C_{r i t} v^{t} D_{j}^{r} \\
& -2 C_{r j t} v^{t} D_{i}^{r}-2 v_{r} D_{i j}^{r} \\
& -2 \bar{C}_{i j}^{l}\left(2 C_{r l t} v^{t} D^{r}+v_{r} D_{l}^{r}\right) .
\end{aligned}
$$

Proof is complete with the observation that $v^{i}(x)$ is Killing in $\bar{F}^{n}$ if and only if $v_{i \| j}+v_{j \| i}+2 \bar{C}_{i j}^{l} v_{l \| 0}=0$, that is, if and only if (21) holds.

If a vector field $v^{i}(x)$ is Killing in $F^{n}$ and $\bar{F}^{n}$, then, from Theorem 1, (21) holds, which on transvection by $y^{i}$ yields

$$
2 C_{r l t} v^{t} D^{r}+v_{r} D_{l}^{r}=0 .
$$

Equation (21), in view of (26), enables us to state the following.

Corollary 2. If a vector field $v^{i}(x)$ is Killing in $F^{n}$ and $\bar{F}^{n}$, then

$$
C_{r i t} v^{t} D_{j}^{r}+C_{r j t} v^{t} D_{i}^{r}+v_{r} D_{i j}^{r}-M_{i j}^{l} v_{l \mid 0}=0
$$

As another important consequence of Theorem 1, we have the following.

Corollary 3. If a vector field $v^{i}(x)$ is Killing in $F^{n}$ and $\bar{F}^{n}$, then the vector $v_{i}(x, y)$ is orthogonal to the vector $D^{i}(x, y)$.

Proof. As $v^{i}(x)$ is Killing in $F^{n}$ and $\bar{F}^{n}$, (21) holds, which on transvection by $y^{i}$ gives (26). Again Transvection (26) by $y^{j}$, it follows that $v_{r} D^{r}=0$. This proves the result.

\section{Conclusion}

The main purpose of the present paper is to examine the classical approach to the problem of existence of Killing vector fields and study how they vary from point to point and how they are related to Killing vector fields defined on the whole manifold. In this respect, our purpose is similar to that of Shukla and Gupta on the study of projective motion. Actually, there is a more substantial relation of our work to theirs where we proved Theorem 1 as the main result and as its consequences we obtained Corollaries 2 and 3. Since the Killing equation (19) is a necessary and sufficient condition for the transformation (18) to be a motion in $F^{n}$, condition (21) obtained in Theorem 1 may be taken as the necessary and sufficient condition for the vector field $v^{i}(x)$, generating a motion in $F^{n}$, to generate a motion in $\bar{F}^{n}$ as well. It is clear that vector field $v^{i}(x)$, generating an affine motion (resp., projective motion) in $F^{n}$, generates an affine motion (resp., projective motion) in $\bar{F}^{n}$ if condition (21) holds. Our study has applications to link various transformations in $F^{n}$ with the corresponding transformations in $\bar{F}^{n}$.

\section{Conflict of Interests}

The authors declare that there is no conflict of interests regarding the publication of this paper.

\section{References}

[1] M. Hashiguchi, "On conformal transformations of Finsler metrics," Journal of Mathematics of Kyoto University, vol. 16, no. 1, pp. 25-50, 1976.

[2] H. Izumi, "Conformal transformations of Finsler spaces," Tensor, vol. 31, pp. 33-41, 1977.

[3] V. K. Kropina, "On projective two-dimensional Finsler spaces with special metric," Trudy Seminara po Vektornomu $i$ Tenzornomu Analizu, vol. 11, pp. 277-292, 1961.

[4] S. H. Abed, "Conformal $\beta$-changes in Finsler spaces," Proceedings of the Mathematical and Physical Society of Egypt, vol. 86, pp. 79-89, 2008.

[5] S. H. Abed, "Cartan connection associated with a $\beta$-conformal change in Finsler geometry," Tensor, N. S., vol. 70, pp. 146-158, 2008.

[6] C. Shibata, "On invariant tensors of $\beta$-changes of Finsler metrics," Journal of Mathematics of Kyoto University, vol. 24, pp. 163$188,1984$.

[7] U. P. Singh, V. N. John, and B. N. Prasad, "Finsler spaces preserving Killing vector fields," Journal of Mathematical and Physical Sciences, vol. 13, pp. 265-271, 1979. 
[8] P. L. Antonelli, Ed., Handbook of Finsler Geometry, Kluwer Academic Publishers, Dordrecht, The Netherlands, 2003.

[9] H. Rund, The Differential Geometry of Finsler Spaces, SpringerVerlag, Berlin, Germany, 1959.

[10] N. L. Youssef, S. H. Abed, and S. G. Elgendi, "Generalized $\beta$ conformal change of Finsler metrics," International Journal of Geometric Methods in Modern Physics, vol. 7, no. 4, article 565, 2010. 


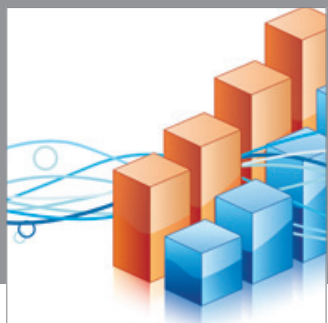

Advances in

Operations Research

mansans

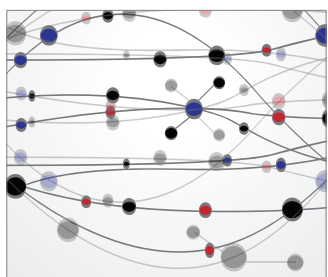

The Scientific World Journal
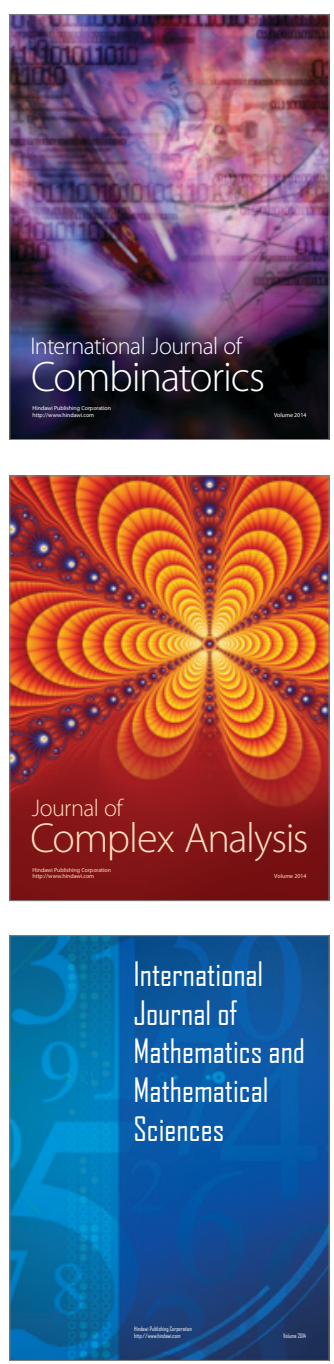
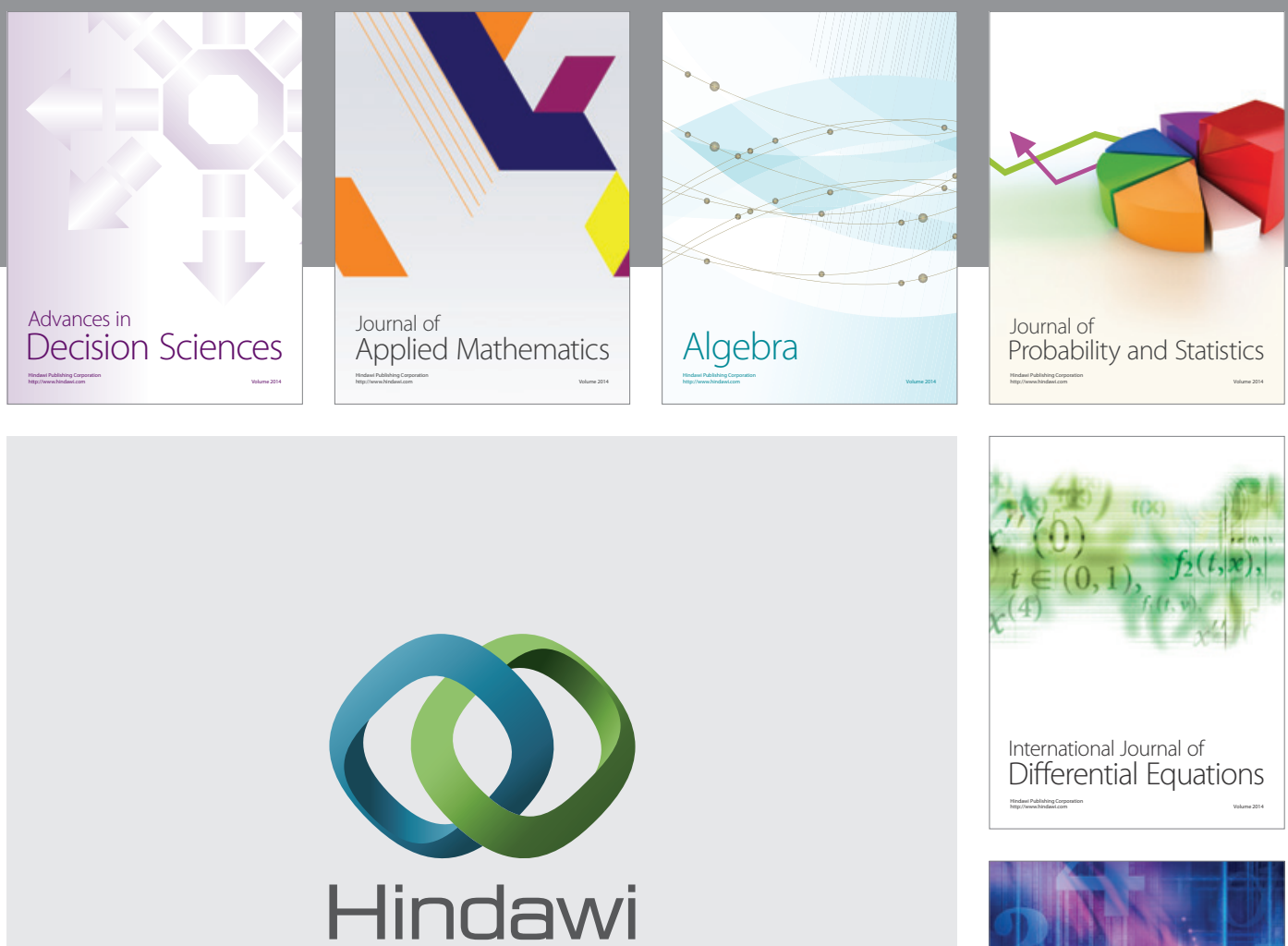

Submit your manuscripts at http://www.hindawi.com
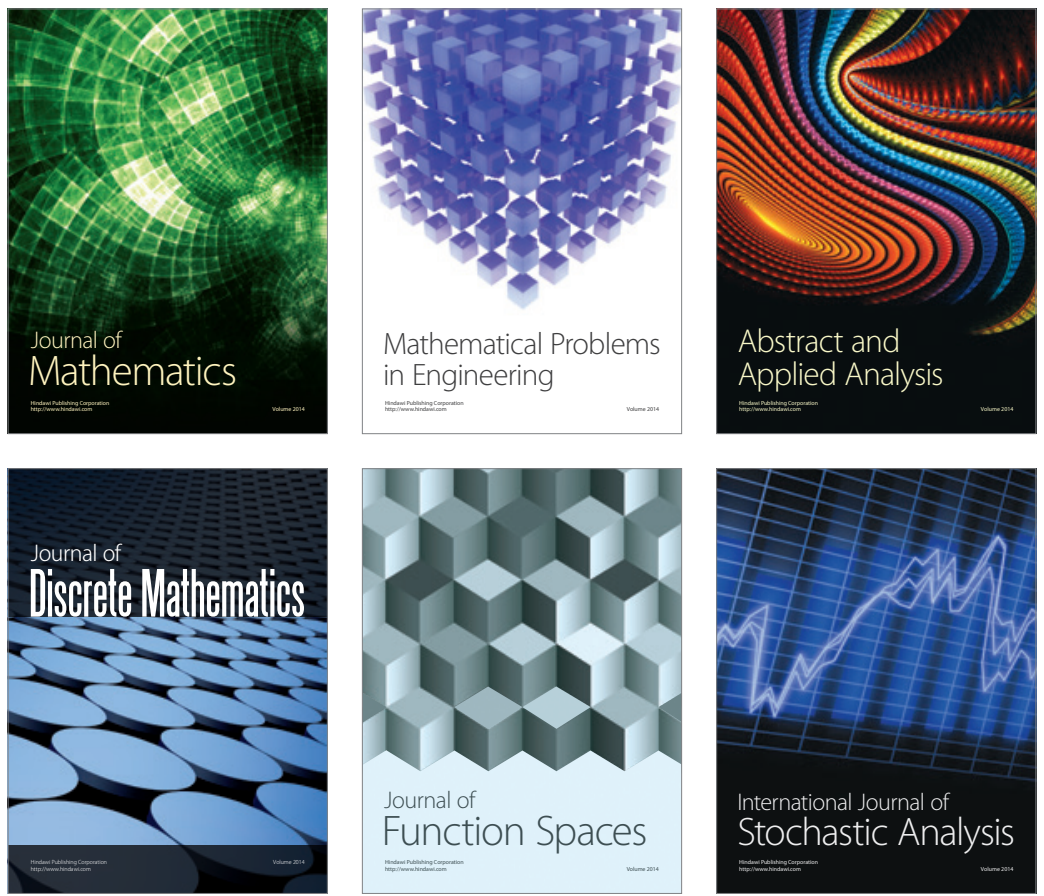

Journal of

Function Spaces

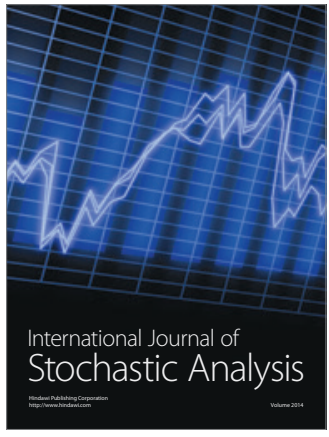

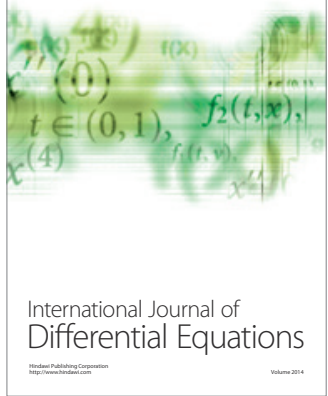
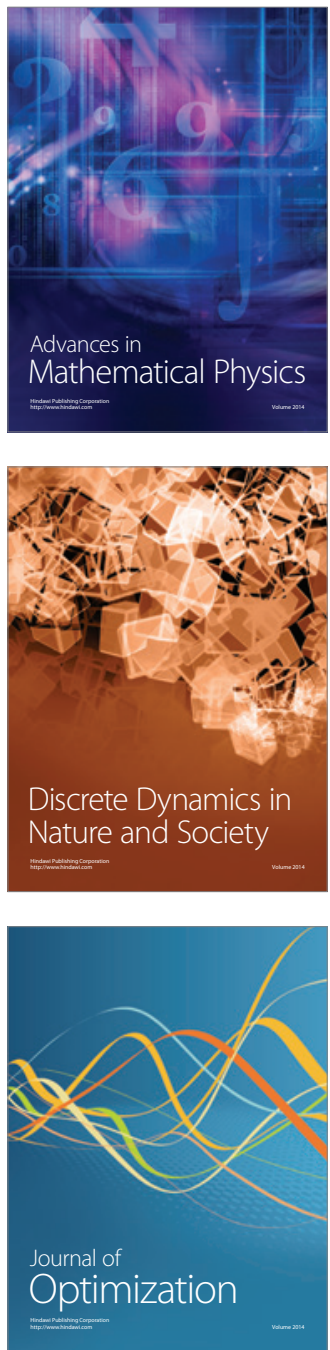